\title{
DIFFERENTIAL DIAGNOSIS OF HYPOECHOIC AND ANECHOIC MASSES WITH GRAY SCALE SONOGRAPHY: NEW OBSERVATIONS
}

\author{
Robert L. Bree, M.D., and Terry M. Silver, M.D.
}

\begin{abstract}
With the technological advances in gray scale sonography that have permitted the use of higher-frequency transducers and expansion of the acoustic dynamic range, increasing problems in differentiating solid masses and fluid-filled masses have become apparent. These difficulties can be overcome by strict adherence to proper scanning techniques, which involve transducer selection, tissue attenuation compensation, and alterations in patient position. The availability of variable-dynamic-range signal processing and the use of real-time scanning can further increase one's confidence in the correct interpretation of these masses. The primary criteria for determining that a mass is fluid-filled have been expanded to include the presence of reverberation echoes, the "lateral shades" sign, and the presence of septations. In the past, hypoechoic masses with low-level internal echoes were termed "complex." Both fluid-filled masses and solid masses may fall into this category. By use of the sonographic criteria, an attempt should be made to determine whether a mass is primarily fluid-filled or solid. Specific anatomic locations and pathologic conditions in which differential diagnosis may be difficult are illustrated; these include abdominal masses, hepatic and renal masses, and pelvic masses.

Indexing Words: Hypoechoic masses - Ultrasound technology - Tissue attenuation compensation - Ultrasound transducers - Abdominal mass - Pelvic mass - Renal mass
\end{abstract}

One of the earliest and most accurate applications of diagnostic ultrasound was to determine the solid or cystic nature of a mass (1-4). Following the development of sophisticated technology and equipment capable of expanding the discernible acoustic dynamic range, emphasis has shifted to imaging solid organs with greater resolution. This newer technology has permitted the routine use of higher-frequency transducers (e.g., 3.5 and $5 \mathrm{MHz}$ ) for abdominal and pelvic ultrasound examinations. Although these transducers markedly improve resolution, their requirement for increased power to the pulser results in a higher level of background noise. This has led to some difficulty in evaluating hypoechoic and anechoic masses (5).

From the Department of Radiology, Toledo Hospital, Toledo, $\mathrm{OH}$, and the University of Michigan Medical Center, Ann Arbor, MI.

Manuscript received December 27, 1978; accepted March 23, 1979.

For reprints contact Robert L. Bree, M.D., Section of Ultrasound and Computed Body Tomography, Department of Diagnostic Radiology, William Beaumont Hospital, 3601 W. 13 Mile Road, Royal Oak, MI 48072.

(C) 1979 by John Wiley \& Sons, Inc.

0091-2751/79/040249-06 \$01.00
An attempt will be made to clarify and expand criteria to aid in the differential diagnosis of hypoechoic and anechoic masses. Scanning techniques, general sonographic concepts, and particular anatomic and pathologic problem areas will be discussed.

\section{SCANNING TECHNIQUES}

Transducer selection. Modern equipment has been developed to permit optimum use of higherfrequency transducers. Selection of the proper transducer for each patient and each examination may be difficult. In general, the highest frequency that will adequately penetrate the area of interest is suggested. Transducer diameter is important when scanning certain anatomic areas, such as between ribs. The larger the diameter in the higher frequencies, the better the resolution for small reflectors, especially at longer focal zones $(5,6)$.

When evaluating hypoechoic masses, the use of transducers of different frequencies is often required for correct diagnosis. Acoustic enhancement is often better evaluated with a higherfrequency transducer (Fig. 1). Masses with high 

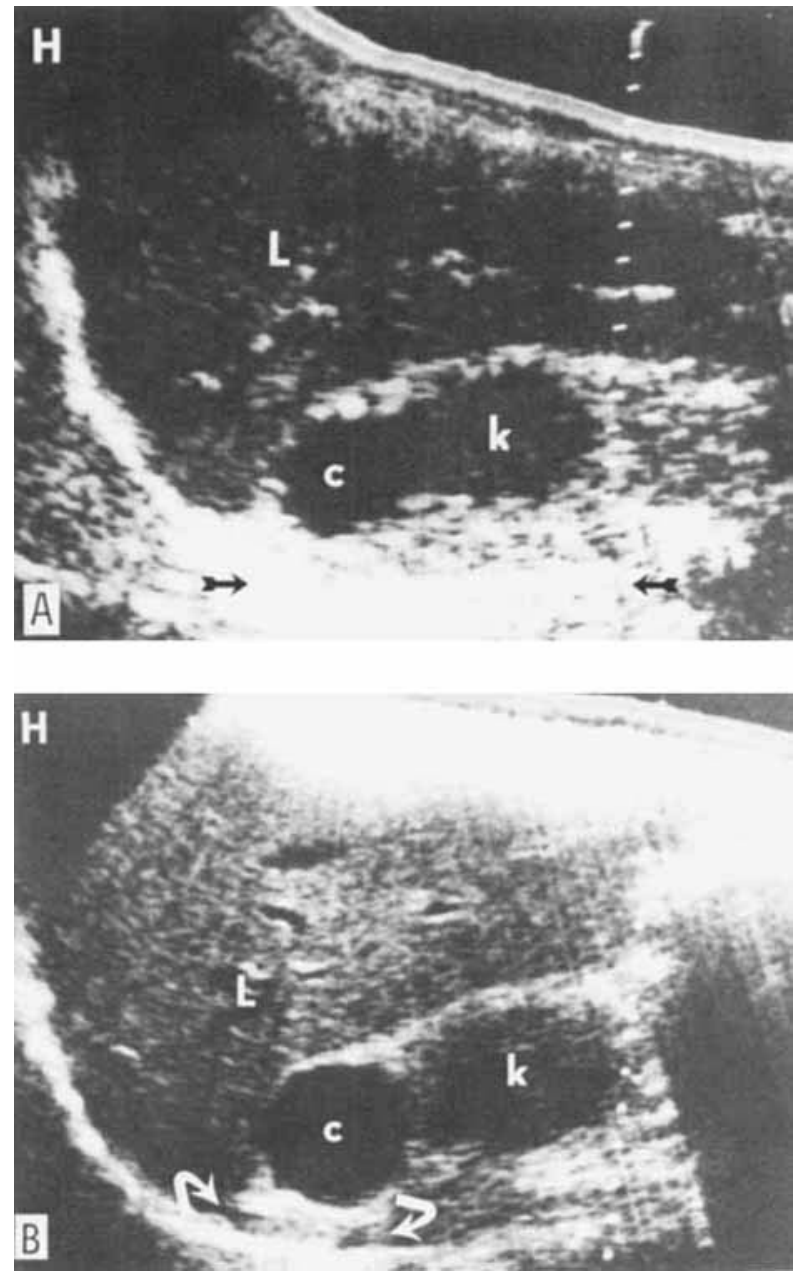

FIGURE 1. Renal cyst, right upper pole. A: Sagittal supine scan with 2.25-MHz transducer. The anechoic zone of the cyst (c) can be seen at the upper pole, but because of the high level of echoes posterior to the entire kidney (arrows), differential acoustic enhancement cannot be appreciated. B: Sagittal supine scan with $3.5-\mathrm{MHz}$ transducer. There is improved resolution of the entire image. The cyst (c) can be easily identified, and the acoustic enhancement (arrows) is much improved. $H$ : direction of patient's head in this and all subsequent scans; k: kidney; L: liver.

attenuation characteristics are best studied with both high- and low-frequency transducers, since attenuation is greater at higher frequencies (Fig. 2). Distinguishing between artifactual echoes and real echoes in a hypoechoic mass is especially difficult when the mass is smaller than $2 \mathrm{~cm}$ (1). In order to make this distinction with confidence, one may have to use a lower-frequency transducer (6) (Fig. 3).

Tissue attenuation compensation. Proper adjustment of the swept gain with different transducers is essential in obtaining diagnostic scans, especially when evaluating hypoechoic masses. An attempt should be made to obtain echo signals of equal amplitude from equal reflectors at all depths of the object being imaged $(3,5,7)$.

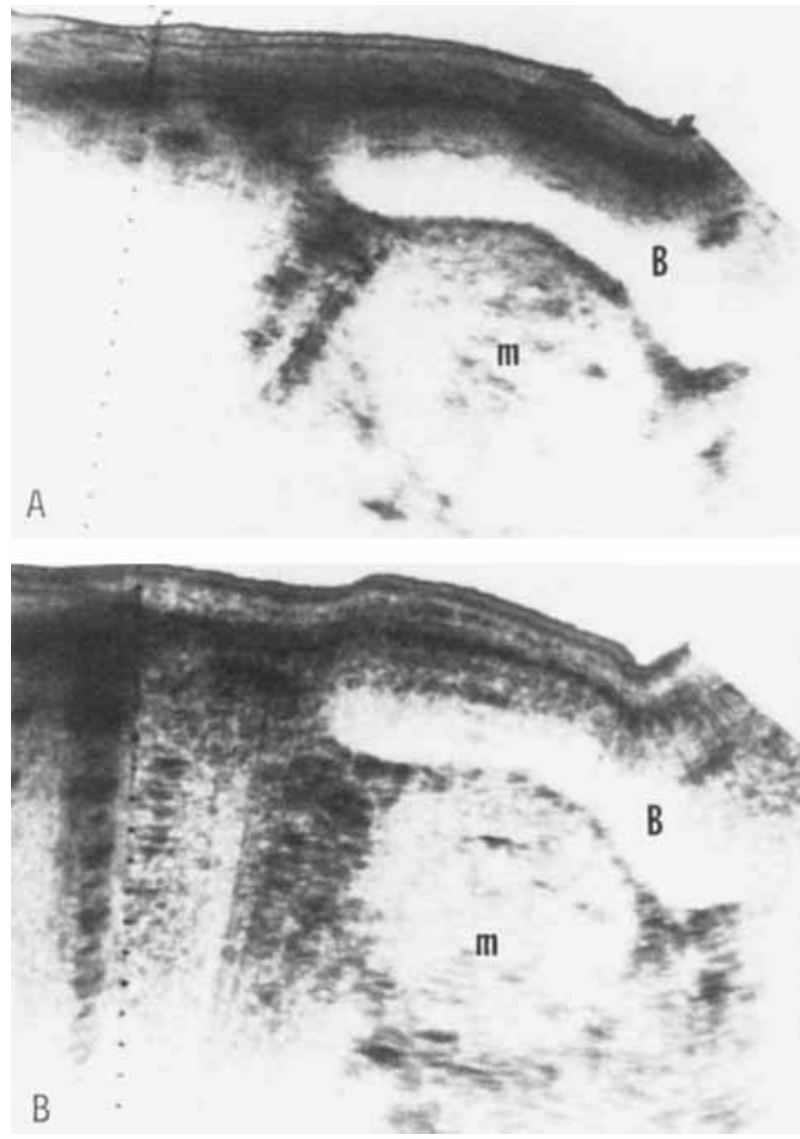

FIGURE 2. Large uterine leiomyoma. A: Sagittal supine scan with 3.5-MHz transducer. At a routine gain and TGC setting there is poor definition of the posterior aspect of the pelvis because of the marked attenuation by the mass (m). B: Sagittal supine scan with $2.25-\mathrm{MHz}$ transducer. The entire mass can now be visualized. The lowerfrequency sound is not attenuated by the homogeneous mass. B: bladder.

Dynamic range of signal processing. In modern gray scale ultrasound displays the echo amplitudes received from the body have a $40-\mathrm{dB}$ range. This range is then compressed into the voltage range to give a full gray scale display. In some cases, varying the range from a full $40 \mathrm{~dB}$ to a narrow 10 or $15 \mathrm{~dB}$ can help distinguish between cystic and solid hypoechoic masses (5).

Patient position. When analyzing hypoechoic and anechoic masses it is important to bear in mind that both the patient's position and the location of the mass can affect the sonographic appearance. Positioning the patient so that the mass is as close as possible to the transducer and is centered perpendicularly over the face of the transducer is helpful when high-frequency transducers are used (8) (Fig. 3). Use of the patient's position to demonstrate acoustic enhancement is important when assessing a mass for cystic characteristics. Acoustic enhancement is possible only when there is an anatomic area into which the sound can travel, as illustrated in Fig. 4. 

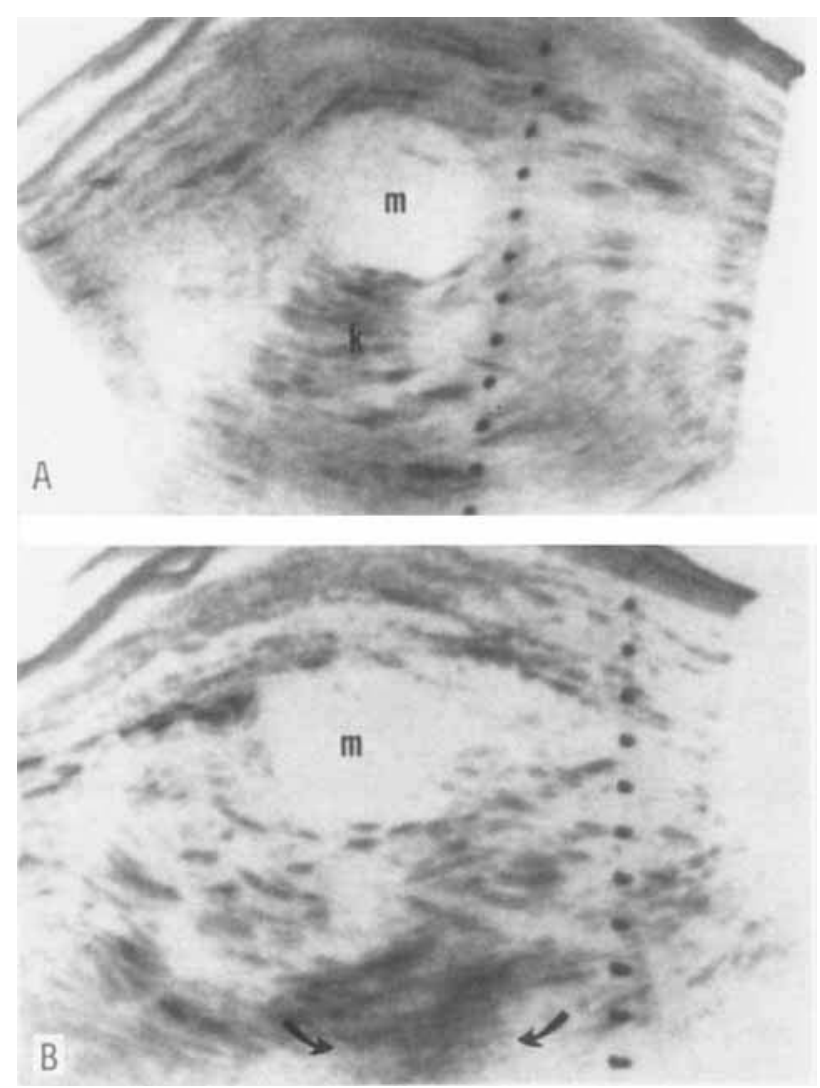

FIGURE 3. Small renal cyst. A: Decubitus scan of the left kidney (k) with $3.5-\mathrm{MHz}$ transducer. A small mass $(\mathrm{m})$ is noted on the lateral aspect of the kidney, with diffuse low-level internal echoes. The use of the decubitus position places the mass closer to the transducer for improved resolution B: Scan in same position with $2.25-\mathrm{MHz}$ transducer. Total absence of echoes can now be appreciated within the mass $(m)$, which represents a renal cyst. By use of a lower-frequency transducer, increased attenuation can be used without sacrificing penetration. Acoustic enhancement beyond the cyst can now be appreciated (arrows).

Real time. With both phased-array and linear-array real-time scanners, artifactual echoes and problems of resolution are common, and they are similar to those encountered with contact scanners. However, the use of real-time scanners does permit easier recognition of acoustic enhancement; this is because the transducer can be held still, and subtle changes in angulation can be made without resorting to compound scanning.

\section{GENERAL SONOGRAPHIC CONCEPTS}

Primary criteria. The classic sonographic criteria for fluid-filled structures have been well described $(1,2,6,9,10)$. These include an absence of echoes, smooth posterior wall margins, acoustic enhancement, and A-mode confirmation (Fig. 4B). Most hypoechoic and anechoic masses meeting these criteria will be purely cystic.

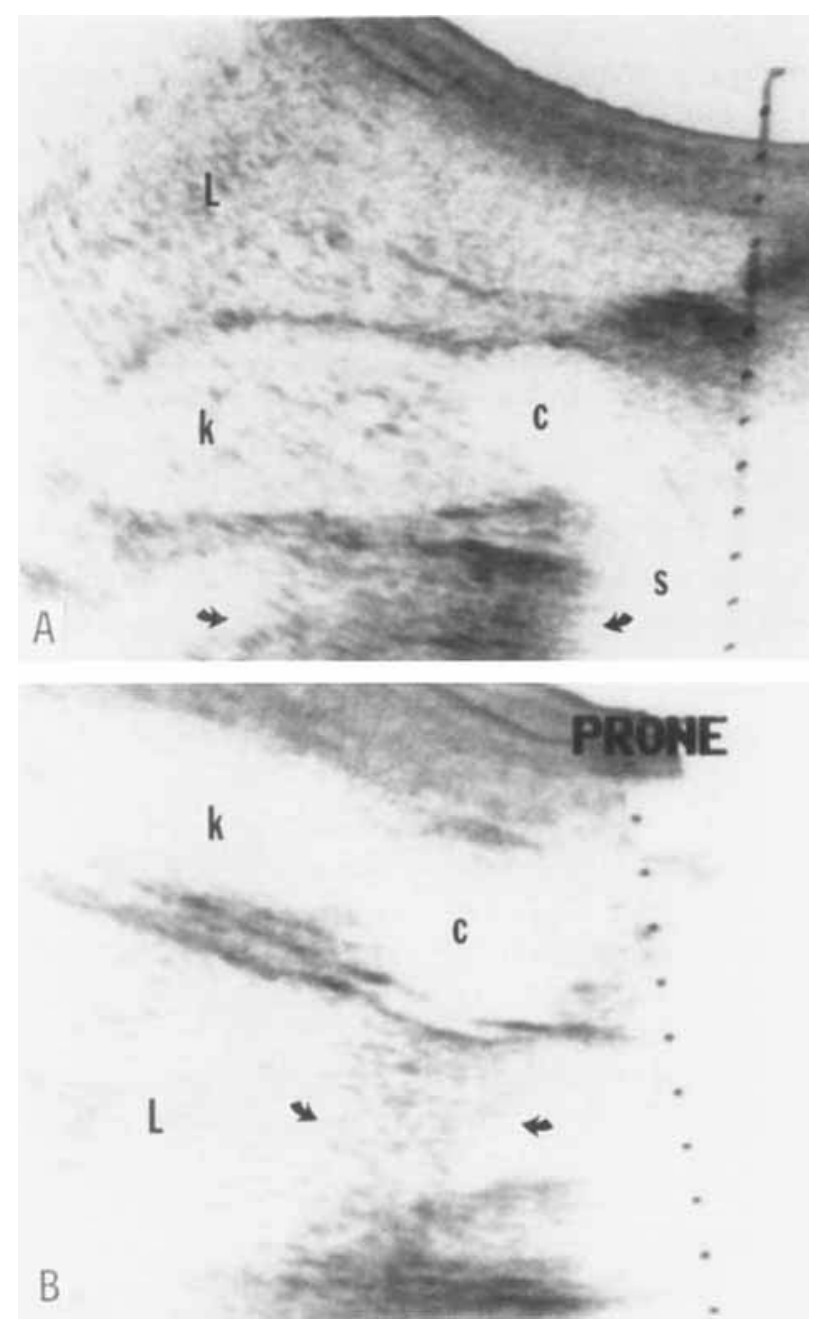

FIGURE 4. Renal cyst, right lower pole. A: Supine scan demonstrates an anechoic mass with cystic characteristics (c). Because of the posterior position of the kidney, the area just beyond it represents a thin layer of muscle and the examining table. There are diffuse echoes (arrows), but it is difficult to identify the acoustic enhancement. Note acoustic shadowing from bowel gas $\langle s\rangle$. B: Prone sagittal scan of right kidney. Since the liver is now just anterior to the kidney, the cyst (c) can be readily seen to exhibit acoustic enhancement in the liver parenchyma (arrows). k: kidney; L: liver.

Secondary criteria. In a small number of cases secondary criteria are necessary to evaluate confusing masses that may be primarily cystic but may contain internal echoes. These echoes may be artifactual or may represent solid components, as in certain cystic masses or necrotic solid masses.

A narrow band of sonolucency can often be visualized posterior to the sides of cystic masses, at the border of the zone of acoustic enhancement. This sign, termed the "lateral shades" sign, when seen in conjunction with acoustic enhancement, is characteristic of fluid; although it can also be seen in association with solid masses, acoustic enhancement will not be present. The sign is caused by refraction of echoes from the wall or capsule of 
the mass and their deflection away from perpendicular transmission. This refraction is caused by the marked change in acoustic impedance between the fluid in the mass and its capsule $(11,12)$ (Fig. 5).

Another sign based on the change in acoustic impedance is the presence of anterior reverberations. This often annoying artifact may prove helpful in distinguishing between cystic and solid masses. Reverberations seen parallel to the path of sound transmission from the transducer are almost always caused by cystic masses. The depth of the reverberations in a cystic mass is the same distance below the strong interface as the interface is below the skin $(5-7)$. On rare occasions reverberations may occur if the tissue anterior to a solid mass has a markedly different acoustic impedance than the mass or if the mass has an unusually thick capsule.

The presence of septations or papillary excrescences projecting into the lumen of an anechoic mass may be taken with confidence as a sign of fluid content. However, these masses may sometimes represent malignant neoplasms (13) (Figs. $6,7)$.

Classification of cystic and solid masses. Most authors divide masses into three major categories: cystic, complex, and solid (14-16). Certain cystic masses such as endometriomas, dermoids, or ovarian cysts with blood or hemorrhagic debris may present with diffuse internal echoes that make it difficult to distinguish them from solid masses without careful evaluation of all ultrasound criteria (15-17) (Fig. 7). Other predominantly cystic masses such as pancreatic pseudocysts and intra-abdominal abscesses or

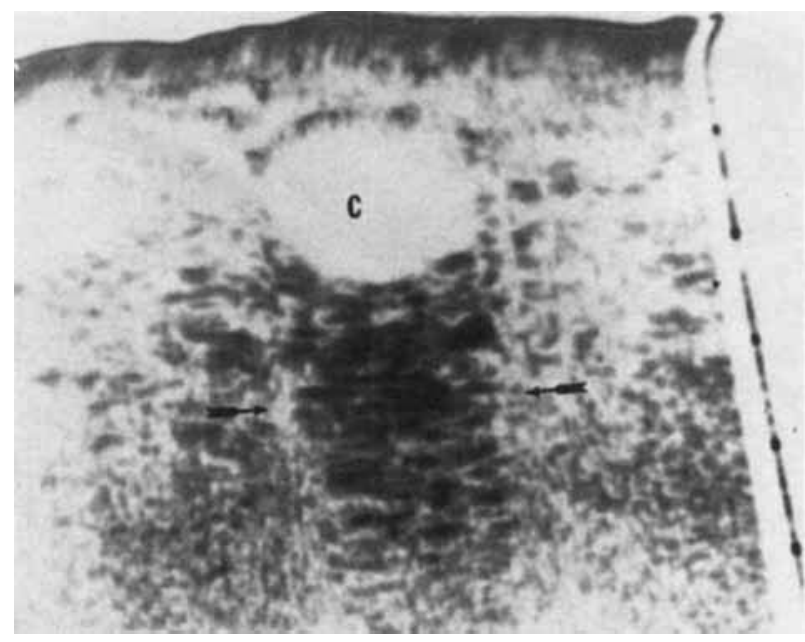

FIGURE 5. Breast cyst with "lateral shades" sign. A sector scan over a breast mass demonstrates an anechoic mass with cystic characteristics (c). There is a narrow band of sonolucency posterior to and on each side of the mass (arrows).

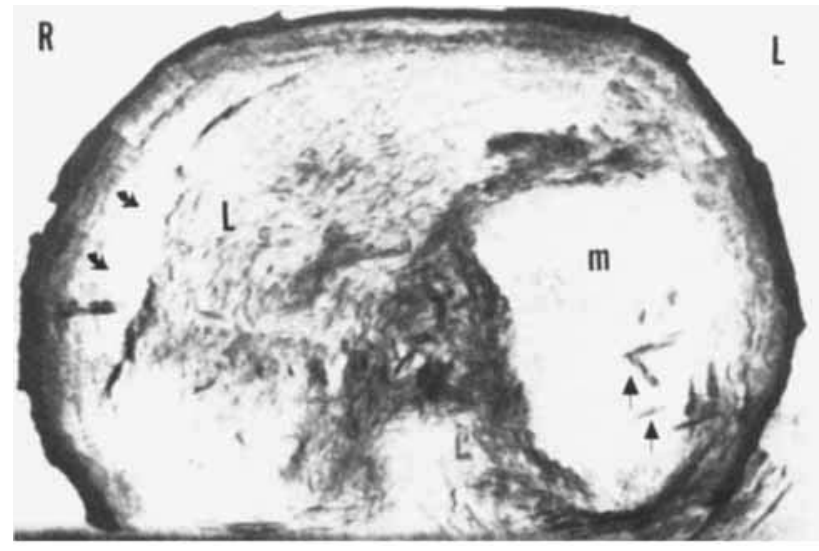

FIGURE 6. Cystadenocarcinoma of pancreas with ascites. Transverse scan in the upper abdomen. A large hypoechoic mass is identified in the region of the pancreatic tail. There are septations within the mass (arrows) indicating fluid content. The low-level echoes indicate floating solid material. Ascites is present (curved arrows) because of extensive peritoneal metastases. At surgery the mass was found to be a fluid-filled but malignant lesion. L: liver; m: mass.

hematomas may also present with diffuse highor low-level echoes $(18,19)$. Some solid masses, particularly sarcomas, hypernephromas, lymphomas, and adenocarcinomas, may be diffusely hypoechoic or may have localized areas of necrosis or fluid $(14,20,21)$ (Figs. 8, 9). The types of masses just described can be termed complex, but such designation adds little to our knowledge of the exact etiology or content of the mass. It is our belief that use of the term complex to describe masses should be avoided. An attempt should be made to determine if a mass is primarily fluidfilled or solid, using the criteria described, and a diagnosis should be made based on this conclusion

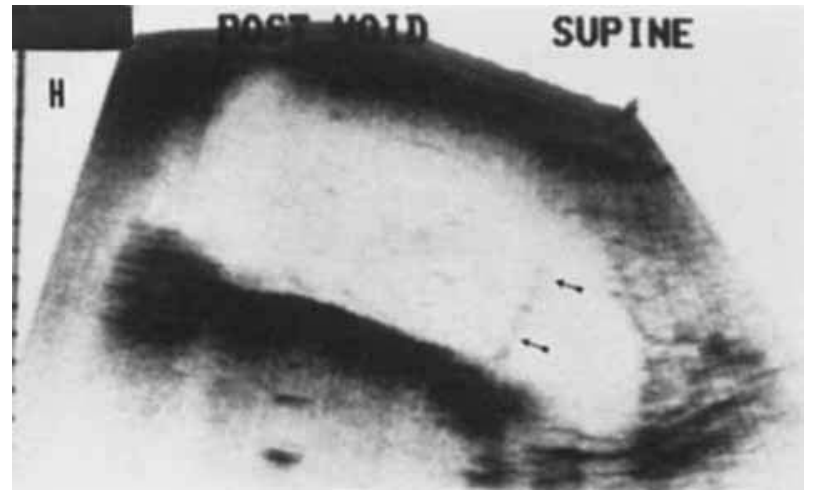

FIGURE 7. Ovarian endometrioma. Supine sagittal scan of the pelvis with empty bladder. A large hypoechoic mass with smooth borders, acoustic enhancement, and anterior reverberations is identified. There is a septum inferiorly (arrows). There are diffuse low-level echoes within the mass that persist with lower-frequency transducers and represent particles from hemorrhage. Knowledge of this phenomenon and the otherwise cystic appearance of the mass led to the correct diagnosis 


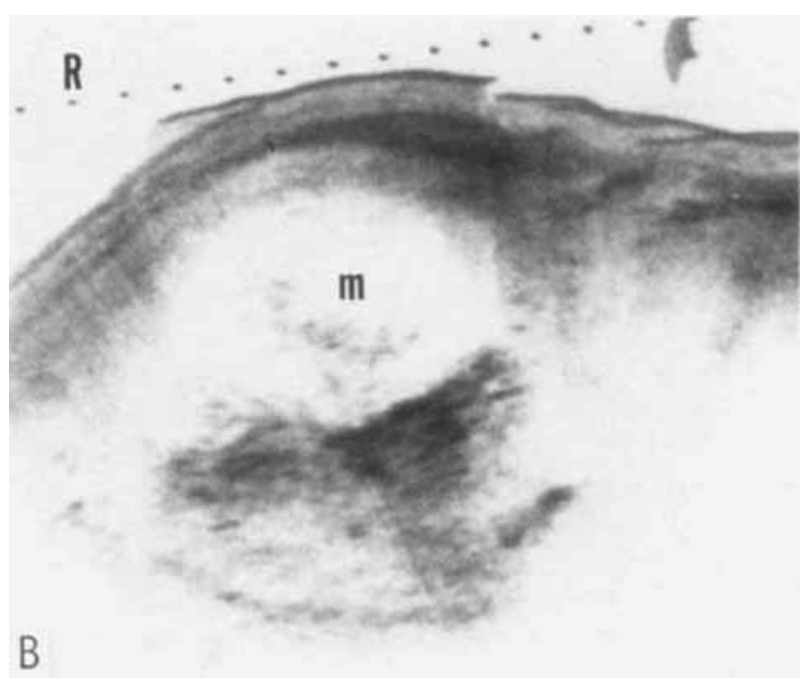

FIGURE 8. Necrotic adenocarcinoma of cecum. Transverse scan in right lower quadrant. A large hypoechoic mass $(\mathrm{m})$ with an irregular margin and low-level internal echoes is shown. There is absence of acoustic enhancement. A large necrotic carcinoma was found at surgery.

plus knowledge of the anatomic location of the mass and the clinical presentation of the patient.

\section{SPECIFIC ANATOMIC AND PATHOLOGIC PROBLEM AREAS}

In addition to general criteria, there are specific anatomic locations and pathologic conditions in which certain criteria are characteristic and may be helpful in determining the etiology of a hypoechoic or anechoic mass.

In the abdomen, lymphomas, adenocarcinomas (Fig. 8), and sarcomas (Fig. 9) may be hypoechoic or anechoic and may contain areas of necrosis $(14,20,22-24)$. Careful evaluation of these masses may be necessary to determine whether they are cystic or solid. Absence of acoustic enhancement most often will suggest that the mass is solid

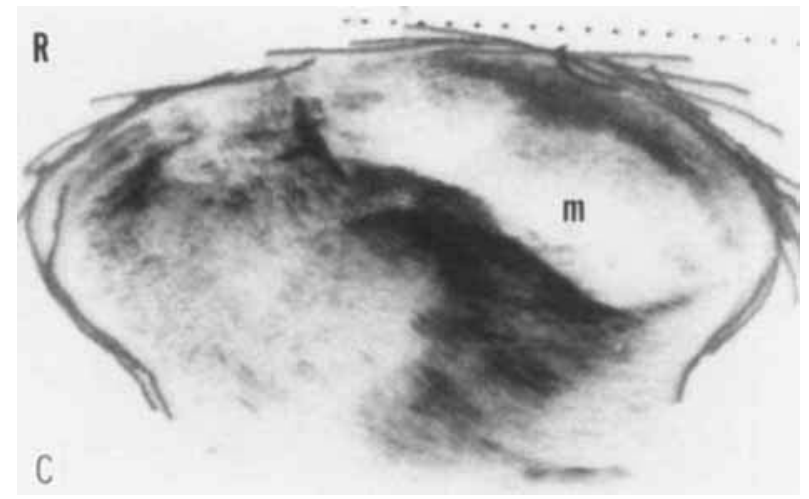

FIGURE 9. Abdominal leiomyosarcoma. Transverse scan in pelvis. A large hypoechoic oval-shaped mass $(\mathrm{m})$ with irregular borders Nonuniform anterior reverberations are present.

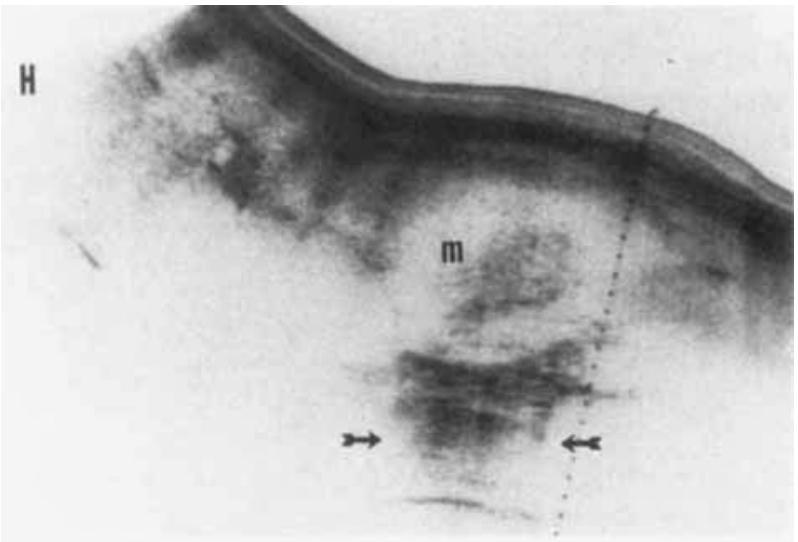

FIGURE 10. Histiocytic lymphoma. Supine sagittal scan of the right side of the abdomen. A large solid mass $(m)$ with high-level internal echoes is seen. There is prominent acoustic enhancement (arrows) even though the mass is solid.

(Figs. 8, 9), but occasionally a solid mass will demonstrate prominent acoustic enhancement (24) (Fig. 10).

In the liver, metastatic sarcomas, adenocarcinomas, and other cell types have been noted to be hypoechoic or anechoic, thus allowing for confusion with hepatic cysts or abscesses. However, in many cases another pattern of neoplasm will be present in the liver, thus confirming the presence of metastatic disease $(21,25)$.

Renal masses may present with confusing sonographic findings. A sign defining hypoechoic or anechoic masses as cystic, as compared with the kidney or other organ to which they are adjacent or are within, is often reliable (3). Since solid renal masses usually contain some internal echoes, and renal neoplasms are very rarely cystic, an anechoic mass in the kidney will be a cyst in more than 90 percent of cases. However, for a confident diagnosis, it is important that the mass satisfy all criteria for a cyst. Overlap of cystic and solid criteria will be especially troublesome in masses measuring $2 \mathrm{~cm}$ or less $(1,10)$.

When evaluating pelvic masses, the bladder and uterus can be used for comparison in distinguishing cystic and solid masses. Hemorrhagic ovarian cysts or endometriomas may contain many internal echoes and may mimic solid masses (15-17) (Fig. 7).

Because of their homogeneity, uterine leiomyomas are often hypoechoic or anechoic, especially with higher-frequency transducers. Such a mass can attenuate sound so much that the far wall of the mass may be difficult to visualize. Use of a lower-frequency transducer will diminish the amount of attenuation and produce further internal echoes. This manipulation can be diagnostic of a uterine leiomyoma (Fig. 2). 
In rare instances hypoechoic or anechoic masses may be encountered that cannot be evaluated correctly with ultrasound. In such cases percutaneous aspiration biopsy techniques with thin needles, using ultrasound guidance for needle placement (26), can be used to make or confirm the correct diagnosis.

\section{REFERENCES}

1. Arger PH, Zarembok I: Source of diagnostic pitfalls in renal sonography. Urology 9:353, 1977.

2. Birnholz JC: Sonic differentiation of cysts and homogeneous solid masses. Radiology 108:699, 1973.

3. Conrad MR, Sanders RC, James AE: The sonolucent "light bulb" sign of fluid collections. J Clin Ultrasound 4:409, 1976.

4. Kossoff G, Garrett WJ, Carpenter DA, et al: Principles and classification of soft tissues by grey scale echography. Ultrasound Med Biol 2:89, 1976.

5. Carson PL: Diagnostic ultrasound-physical principles and equipment, in Waggener RG, Shalek RJ (eds): Handbook of Medical Physics. Cleveland, CRC Press, in press.

6. Bartrum RJ, Crow HC: Gray Scale Ultrasound. Philadelphia, WB Saunders, 1977.

7. Skolnick ML, Meire HB, Lacky JW: Common artifacts in ultrasound scanning. $\mathrm{J}$ Clin Ultrasound 3:273, 1975.

8. Albarelli JN, Lawson TL: Renal ultrasonography: Advantages of the decubitus position. J Clin Ultrasound 6:115, 1978.

9. Carlsen EN: Ultrasound physics for the physician: A brief review. J Clin Ultrasound 3:69, 1975.

10. Green WM, King DL, Casarella WJ: A reappraisal of sonolucent renal masses. Radiology 121:163, 1976.

11. Jellins J, Kossoff G, Reeve TS: Detection and classification of liquid-filled masses in the breast by gray scale echography. Radiology 122:207, 1977.

12. Kobayashi T: Gray-scale echography for breast cancer. Radiology 122:207, 1977.
13. Wolson AH, Walls WJ: Ultrasonic characteristics of cystadenoma of the pancreas. Radiology 119:203, 1976.

14. Goldberg BB: Ultrasonic evaluation of superficial masses. J Clin Ultrasound 3:91, 1975.

15. Lawson TL, Albarelli JN: Diagnosis of gynecologic pelvic masses by gray scale ultrasonography: Analysis of specificity and accuracy. Am J Roentgenol 128:1003, 1977.

16. Sandler MA, Karo JJ: The spectrum of ultrasonic findings in endometriosis. Radiology 127:229, 1978.

17. Cunningham JJ, Wooten $\mathrm{W}$, Cunningham MA: Gray scale echography of soluble protein and protein aggregate fluid collections (in vitro study). J Clin Ultrasound 4:417, 1976.

18. Doust BD, Quiroz F, Steward JM: Ultrasonic distinction of abscesses from other intra-abdominal fluid collections. Radiology 125:213, 1977.

19. Sokoloff J, Gosink BB, Leopold GR, et al: Pitfalls in the echographic evaluation of pancreatic disease. J Clin Ultrasound 2:321, 1974.

20. Bree RL, Green B: The gray scale sonographic appearance of intra-abdominal mesenchymal sarcomas. Radiology 128:193, 1978.

21. Wooten W, Green B, Goldstein HM: Ultrasonography of necrotic hepatic metastases. Radiology 128:447, 1978.

22. Filly RA, Marglin S, Castellino RA: The ultrasonographic spectrum of abdominal and pelvic Hodgkin's disease and non-Hodgkin's lymphoma. Cancer 38:2143, 1976.

23. Freimanis AK: Ultrasonic imaging of neoplasms. Cancer 37:496, 1976 .

24. Yeh HC, Wolf BS: Ultrasonography and computed tomography in the diagnosis of homogeneous masses. Radiology 123:425, 1977.

25. Green B, Bree RL, Goldstein HM, et al: Gray scale ultrasound evaluation of hepatic neoplasms: Patterns and correlations. Radiology 124:203, 1977.

26. Goldstein HM, Zornosa J, Wallace S, et al: Percutaneous fine needle aspiration biopsy of pancreatic and other abdominal masses. Radiology 123:319, 1977. 